\title{
CAN AUSTROPOTAMOBIUS ITALICUS MERIDIONALIS BE USED AS A MONITORING INSTRUMENT IN CENTRAL ITALY? PRELIMINARY OBSERVATIONS
}

\author{
M. SCALICI (1), G. GIBERTINI (2).
}

Università degli Studi "Roma Tre", Dipartimento di Biologia, viale G. Marconi 446, 00146 Roma, Italy.

(1) E-mail: scalici@uniroma3.it

(2) E-Mail: gibertin@uniroma3.it

Reçu le 5 novembre 2004

Accepté le 11 Avril 2005

Received November 5, 2004

Accepted April 11, 2005

\begin{abstract}
Attributing the term bioindicator to a species complex such as Austropotamobius pallipes is complicated, considering that the meaning given to a bioindicator can vary according to the geographic position, and that $A$. pallipes species complex has been divided into 2 species and 5 subspecies, the possibility of using $A$. i. meridionalis as a bioindicator in watercourses of Central Italy was evaluated. To this end, we analysed the structure and abundance of a number of crayfish populations and then compared them to the water quality of the corresponding watercourses, using E.B.I. (Extended Biotic Index). This index provides information on water quality through an analysis of the macrobenthos community. The watercourses are assigned to various classes (from I to V, increasing the degrees of alteration). This study was carried out in several watercourses near Rome. The abundance of crayfish was found to be greatest in those tracts that are assessed as class I, where the populations seem well structured with individuals of various sizes and the sex ratio is approximately $1: 1$. In contrast, in class II we found a reduced presence of specimens, mostly in bad conditions. No crayfish specimens were observed in watercourses designated as class III. In light of these preliminary results, it does not seem possible to consider $A$. $i$. meridionalis as a bioindicator, given that one of the typical characteristics of a biological indicator (wide distribution) is not met. Though these results illustrate the possibility of using $A$. i. meridionalis populations as an excellent monitoring instrument in Central Italy.
\end{abstract}

Key-words: Austropotamobius italicus meridionalis, bioindicator, monitoring instrument, water quality.

\section{AUSTROPOTAMOBIUS ITALICUS MERIDIONALIS PEUT-ELLE ÊTRE UTILISÉE COMME INSTRUMENT DE SURVEILLANCE EN ITALIE DU CENTRE? OBSERVATIONS PRÉLIMINAIRES}

\section{RÉSUMÉ}

II est très compliqué d'attribuer le terme de bio-indicateur à une espèce complexe comme Austropotamobius pallipes, considérant que le sens attribué à «bio-indicateur » 
peut changer selon la région et que l'ensemble d'espèces Austropotamobius pallipes a été fractionné en deux espèces et cinq sous-espèces. On a cependant évalué la possibilité d'utiliser A. i. m. comme bio-indicateur dans les cours d'eau d'Italie Centrale. La structure et l'abondance des populations d'écrevisses furent évaluées et ensuite confrontées à la qualité des cours d'eau correspondants, en utilisant l'I.B.E. (Index Biotique Étendu), index qui donne des informations sur la qualité de l'eau à travers l'analyse de la communauté macrobenthique. Les cours d'eau sont classés selon leur qualité (de I jusqu'à V, selon des degrés d'altération croissant). Cette recherche fut réalisée sur différents cours d'eau près de Rome. Les écrevisses furent trouvées en plus grande abondance dans les sections de cours d'eau appartenant à la classe I de l'I.B.E., où les populations paraissent bien structurées avec des spécimens de tailles variées et où le sex-ratio est à peu prés $1: 1$. On a au contraire trouvé peu de spécimens dans les cours d'eau de la classe II, et ils étaient en mauvais état sanitaire. On n'a trouvé aucune écrevisse dans les cours d'eau de la classe III. Selon ces études préliminaires, il n'est pas possible de considérer A. i. $m$. comme bio-indicateur, vu que l'une des caractéristiques typiques d'un indicateur biologique (large distribution) n'est pas retrouvée ici, bien que ces résultats démontrent que le suivi des populations d'écrevisses est un excellent moyen de surveillance des cours d'eau en Italie Centrale.

Mots-clés : Austropotamobius italicus meridionalis, bioindicateur, instrument de surveillance, qualité de l'eau.

\section{INTRODUCTION}

The distribution of the white-clawed freshwater crayfish, Austropotamobius pallipes species complex, covers Western Europe from $56^{\circ} \mathrm{N}$ in Great Britain to $38^{\circ} \mathrm{S}$ in Spain, and from $8^{\circ} \mathrm{W}$ in Ireland to $20^{\circ} \mathrm{E}$ in Yugoslavia (LAURENT, 1988). Its distribution seems to be limited primarily by calcium concentration, requiring $2.8 \mathrm{mg} / \mathrm{l}$ for calcification of the exoskeleton (CHASEMARTIN, 1967). Crayfishes are important in trophic dynamics of freshwater, particularly as macrophyte grazers and detrital feeders (MOMOT, 1995; COVICH et al., 1999; SCALICl et al., 2004).

Since the 1970s, this species has drastically decreased its geographical range, mainly due to a combination of factors including over-fishing, habitat modification, such as pollution (particularly form farm - FOSTER and TURNER, 1993), habitat destruction (WESTMAN, 1985; HOLDICH and LOWERY, 1988; HOLDICH et al., 1999), engineering works (LOWERY and HOGGER, 1986), river canalisation (HOGGER and LOWERY, 1982), introduction of alien crayfishes (GHERARDI and HOLDICH, 1999) and crayfish plague (ALDERMANN and POLGASE, 1988). A. pallipes is listed as an endangered species by the IUCN (GROOMBRIDGE, 1993), in Appendix III of the Bern Convention and in Annex II and $V$ of the Directive 92/43/ECC.

Several authors have analysed the systematics of the $A$. pallipes species complex (GRANDJEAN et al., 1998; GRANDJEAN et al., 2000b; LARGIARDER et al., 2000). This species was recently divided in two taxa, Austropotamobius pallipes, with only the nominal subspecies, and A. italicus with four subspecies: italicus, carsicus, carinthiacus and meridionalis (GRANDJEAN et al., 2002a-b; FRATINI et al., 2005). Following this scheme, it is necessary to include $A$. italicus in the Invertebrate Red Data Book as a species distinct from $A$. pallipes. Actually, in Latium, the species is preserved by the regional law $n^{\circ} 88 / 18$, which forbids catch, transport and sale.

A. italicus is the unique autochthonous species in Central Italy. Especially in Latium, it shows a fragmented distribution, with very isolated freshwater crayfish stocks (SCALICI, 2004). A. italicus lives in cold and oligotrophic lotic waters with a high concentration of dissolved oxygen. For its ecological characteristics, the white-clawed crayfish has 
been described as sensitive to pollution and thus considered as a potential water quality bioindicator (HOLDICH and REEVE, 1991; REYNOLDS et al., 2002). Despite the progressive worsening of water quality, there are still suitable conditions in Latium to study the white-clawed freshwater crayfish. Information on the quality of all watercourses in this region is not available, but it is generally considered to be low (MANCINI and ARCÁ, 2000) due to increasing eutrophication.

The aim of this study is to describe the ecological requirements (water quality) of $A$. italicus, in Central Italy, in order to explain its current restricted and fragmented distribution. From July 2003 to April 2004, a survey was conducted to assess the status of the freshwater crayfish stocks in Latium and to evaluate water quality.

\section{MATERIAL AND METHODS}

\section{Study area}

This research was carried out in different watercourses of the Lucretili, Simbruini, Carseolani, and Sabini Mountains, located between the Provinces of Rome and Rieti and belonging to the Tiber River basin (Figure 1). The study area ranges from $42^{\circ} 20^{\prime} \mathrm{N}$ to $42^{\circ} \mathrm{S}$ and from $12^{\circ} 40^{\prime} \mathrm{W}$ to $13^{\circ} 10^{\prime} \mathrm{E}$ and it is located on the carbonate platform of Latium and Abruzzi. The altitude of the monitored watercourses ranged from a minimum of $452 \mathrm{~m}$ a. s.l. (River Duranna) to a maximum of $640 \mathrm{~m}$ a.s.l. (River Ariana). Using the systematic proposed by FRATINI et al. (2005), Austropotamobius italicus meridionalis is the endemic subspecies present (unpublished data) within the study area.

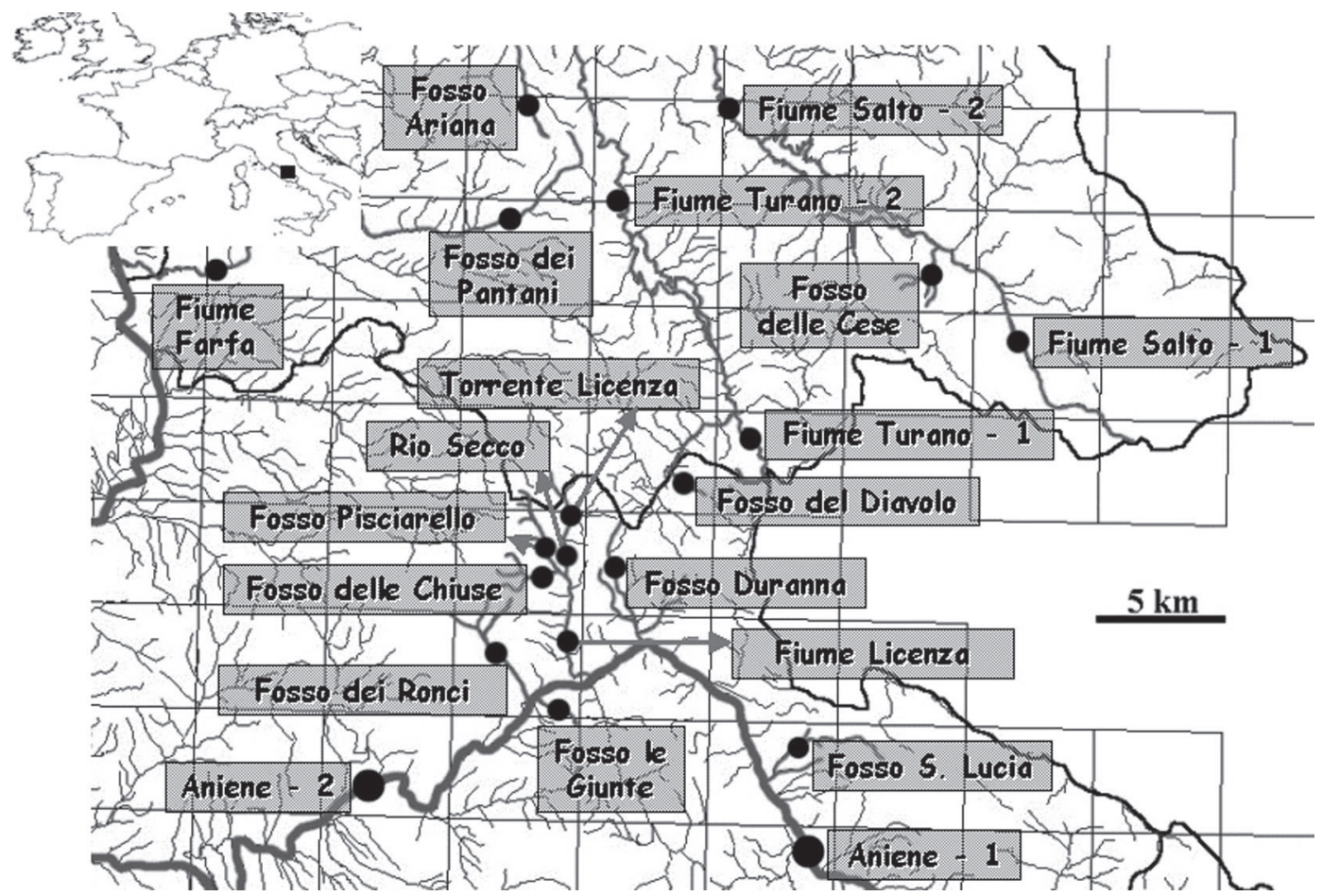

Figure 1

Location of the sampled brooks.

Figure 1

Localisation des cours d'eau étudiés. 


\section{Table I}

Classification of the watercourses in quality classes of the E.B.I. (Extended Biotic Index). The values of this biotic index are determined observing the presence/ absence of the genus or family of the different invertebrate groups.

Tableau I

Classification des cours d'eau en classes de qualité de l'I.B.E. (Index Biotique Étendu). Les valeurs de cet index biotique ont étés déterminées en observant la présence/absence du genre ou de la famille de différents groupes invertébrés.

\begin{tabular}{ccl}
\hline $\begin{array}{c}\text { Quality } \\
\text { class }\end{array}$ & $\begin{array}{c}\text { E.B.I. } \\
\text { value }\end{array}$ & \multicolumn{1}{c}{$\begin{array}{c}\text { Quality } \\
\text { evaluation }\end{array}$} \\
\hline I & $10-15$ & $\begin{array}{l}\text { unpolluted environment or not deteriorated to a } \\
\text { considerable extent; }\end{array}$ \\
\hline II & $8-9$ & $\begin{array}{l}\text { environment with moderate symptoms of pollution or } \\
\text { deterioration; }\end{array}$ \\
\hline III & $6-7$ & polluted or deteriorated environment; \\
\hline IV & $4-5$ & very polluted or deteriorated environment; \\
\hline V & $1-3$ & extremely polluted or deteriorated environment. \\
\hline
\end{tabular}

\section{Sampling procedure}

The research was carried out in July and October 2003, and January and April 2004. Each month the sampling phase lasted 14 days, the minimal time necessary to investigate all sites. Each site was sampled four times. Every site ranged from 150 to $180 \mathrm{~m}$ in length. The average width and depth as well as the presence of aquatic and riparian vegetation were recorded and observed for every site. Moreover, physicochemical characteristics were measured, including temperature, $\mathrm{pH}$, conductivity and oxygen concentration, using a digital meter with appropriate probes for immersion (Multiline F/SET-3), and ammonium, nitrites, nitrates, total phosphate concentration and total hardness were recorded using a spectrophotometer (Photometer MPM 3000). Subsequently, macrobenthos collections were carried out using a standard hand net, with a $25 \times 19 \mathrm{~cm}$ rectangular chassis supporting a $0.4 \times 0.5 \mathrm{~mm}$ mesh net. At the end of the net, a plastic cylinder collected the macrobenthos organisms. The standard hand net was positioned with its open end against the stream current, and the outer rim on the bottom; stones were moved with hands or by kicking. This procedure was carried out along a diagonal transect from the left to the right side of the watercourse. Three invertebrate samples were collected for each site. All invertebrates were preserved in $70 \%$ alcohol and identified in the laboratory using a macroinvertebrates guide (CAMPAIOLI et al., 1994, 1998). Thus, the E.B.I. (Extended Biotic Index) was calculated in order to attribute a water quality value to the monitored watercourses. It is the unique legal biotic index in Italy according to the national norms concerning preservation of the inland water by pollution (D.L. 99/152). By applying the E.B.I., it is possible to obtain values varying from 1 to 15 . The watercourses are assigned to various classes (from I to V, increasing degrees of alteration - Table I). Subsequent to the macroinvertebrate sampling, white-clawed freshwater crayfish were caught, sexed, weighed (to the nearest $0.01 \mathrm{~g}$ ) and post-orbital length (POL, from the posterior orbital edge to the posterior dorsal extremity of the cephalothorax - according to GRANDJEAN et al., 1997) was measured to the nearest $0.05 \mathrm{~mm}$ using an eyepiece micrometer. Crayfish sampling was done during the twilight, and each watercourse was monitored two times: once upstream, and once downstream. CPUE (catch per unit effort) and the relative abundance of crayfish were described. CPUE represented the total number of crayfish 
caught divided by the time spent sampling (according to DEMERS and REYNOLDS, 2002), which lasted a standard of 100-120 minutes. Instead, the relative abundance was obtained calculating the biomass according to the following formula:

$$
A=W_{h} \times B_{h}
$$

where $A$ is the weighted average of the total weight of the samplings, $W_{h}$ represents the portion of the monitored area (measured in $\mathrm{m}^{2}$ ) where freshwater crayfish occurred divided by the total area of the investigated tract and $B_{h}$ is the average of the total weight measured in each site (SPEDICATO and CANNAS, 2000).

\section{RESULTS}

\section{Habitat features}

During the research period, the values of chemical and physical parameters had the same range and similar trends in different sites. Average values are shown in Table II.

$\mathrm{pH}(7-8)$ and total hardness (72-84 $\mathrm{mg} / \mathrm{l})$ values were constant in each site while $\mathrm{NO}_{3}{ }^{-}$and total phosphate were the more variable parameters.

\section{Table II}

Summary of chemical, physical and morphological measurements of every site.

\section{Tableau II}

Résumé des mesures chimiques, physiques et morphologiques des sites.

\begin{tabular}{lcccccccc}
\hline & $\begin{array}{c}\mathbf{T} \\
\left({ }^{\circ} \mathbf{C}\right)\end{array}$ & $\begin{array}{c}\mathbf{O}_{\mathbf{2}} \\
(\mathbf{m g} / \mathbf{l})\end{array}$ & $\begin{array}{c}\mathbf{N H}_{\mathbf{4}}{ }^{+} \\
(\mathbf{m g} / \mathbf{l})\end{array}$ & $\begin{array}{c}\mathbf{N O}^{-} \\
(\mathbf{m g} / \mathbf{l})\end{array}$ & $\begin{array}{c}\mathbf{N O}_{3}{ }^{-} \\
(\mathbf{m g} / \mathbf{l})\end{array}$ & $\begin{array}{c}\text { TP } \\
(\mathbf{m g} / \mathbf{l})\end{array}$ & $\begin{array}{c}\text { Width } \\
(\mathbf{m})\end{array}$ & $\begin{array}{c}\text { Depth } \\
(\mathbf{m})\end{array}$ \\
\hline T. Licenza & 12.8 & 5.1 & $<0.5$ & $<0.05$ & 0.018 & 0.069 & 3 & 1.8 \\
\hline F. Pisciarello & 12.9 & 4.8 & $<0.5$ & $<0.05$ & 0.02 & 0.06 & 1.8 & 1.5 \\
\hline F. Duranna & 12.3 & 4.6 & $<0.5$ & 0.058 & 0.025 & 0.056 & 2 & 1.4 \\
\hline F. le Giunte & 12.2 & 4.9 & $<0.5$ & $<0.05$ & 0.029 & 0.049 & 2.5 & 1.6 \\
\hline F. S. Lucia & 11.2 & 5 & $<0.5$ & $<0.05$ & 0.03 & 0.04 & 2.8 & 1.7 \\
\hline F. delle Cese & 12.1 & 5.3 & $<0.5$ & $<0.05$ & 0.014 & 0.038 & 3 & 1.7 \\
\hline F. del Diavolo & 11.7 & 5.1 & $<0.5$ & $<0.05$ & 0.017 & 0.059 & 3.1 & 1.9 \\
\hline F. Ariana & 12 & 5.9 & $<0.5$ & $<0.05$ & 0.03 & 0.054 & 2.4 & 1.9 \\
\hline F. dei Pantani & 13 & 6.1 & $<0.5$ & $<0.05$ & 0.025 & 0.048 & 2.7 & 1.8 \\
\hline F. dei Ronci & 13.3 & 5.4 & $<0.5$ & $<0.05$ & 0.024 & 0.041 & 3 & 1.6 \\
\hline F. Farfa & 15.2 & 3.8 & 0.6 & $<0.05$ & 0.054 & 0.089 & 7.4 & 4.2 \\
\hline Rio Secco & 12.4 & 4.8 & 0.5 & $<0.05$ & 0.07 & 0.01 & 2.5 & 2 \\
\hline F. delle Chiuse & 12.7 & 3.7 & 0.5 & $<0.05$ & 0.08 & 0.05 & 2.4 & 3.1 \\
\hline Aniene - 1 & 15.4 & 3.4 & 0.7 & 0.06 & 0.1 & 0.02 & 4.5 & 2.6 \\
\hline F. Turano - 1 & 15.3 & 3.9 & 0.6 & 0.08 & 0.24 & 0.034 & 3.5 & 3.2 \\
\hline F. Salto - 1 & 15.1 & 3.8 & 0.7 & 0.078 & 0.38 & 0.083 & 3.5 & 2.5 \\
\hline F. Salto - 2 & 15.8 & 3.6 & 0.7 & 0.064 & 0.78 & 0.1 & 4 & 2 \\
\hline F. Licenza & 14.2 & 2.8 & 1.2 & 0.089 & 1 & 0.34 & 3.5 & 2.8 \\
\hline Aniene - 2 & 16.1 & 2.8 & 0.9 & 0.078 & 0.87 & 0.5 & 5 & 3.6 \\
\hline F. Turano - & 15.9 & 3 & 1 & 0.085 & 1.2 & 0.48 & 4.5 & 3 \\
\hline & & & & & & & & \\
\hline
\end{tabular}




\begin{tabular}{|c|c|c|c|c|c|c|c|c|c|c|c|c|c|c|c|c|c|c|c|c|c|}
\hline & & 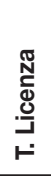 & 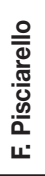 & 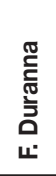 & 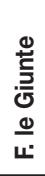 & $\begin{array}{l}\frac{\pi}{0} \\
3 \\
\\
\text { ம் } \\
4\end{array}$ & 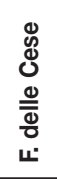 & 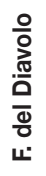 & 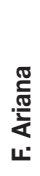 & 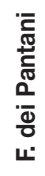 & 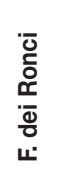 & $\begin{array}{l}\frac{\pi}{5} \\
\frac{\pi}{\varpi} \\
\text { एँ }\end{array}$ & 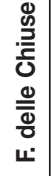 & $\begin{array}{l}\stackrel{0}{0} \\
\mathbb{J} \\
\stackrel{0}{\circ} \\
\stackrel{\circ}{x}\end{array}$ & 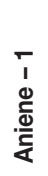 & 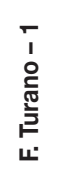 & 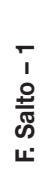 & 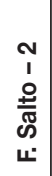 & 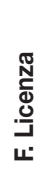 & 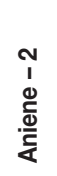 & 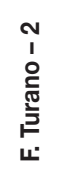 \\
\hline \multicolumn{22}{|l|}{ Plecoptera } \\
\hline Nemouridae & Protonemura & - & - & 18 & 32 & 23 & 15 & 8 & 11 & 9 & 7 & 22 & 1 & - & - & - & - & - & - & - & - \\
\hline & $A$ & 22 & 19 & - & - & - & 16 & 15 & 12 & 9 & 15 & 34 & 4 & - & - & - & - & - & - & - & - \\
\hline Perlodidae & Isoperla & 20 & 24 & 16 & 31 & 24 & - & 24 & 15 & - & - & 19 & 2 & - & - & - & - & - & - & - & - \\
\hline Leuctridae & Leuctra & 24 & 8 & - & 12 & 13 & 9 & 12 & - & 7 & 5 & - & 12 & - & 16 & 4 & - & 7 & - & - & - \\
\hline \multicolumn{22}{|l|}{ Ephemeroptera } \\
\hline \multirow[t]{3}{*}{ Baetidae } & Baetis & 6 & 72 & 94 & 93 & 4 & 4 & 19 & - & 4 & 13 & 66 & - & 74 & - & 82 & - & 15 & 244 & 115 & - \\
\hline & Caenis & 18 & 44 & 32 & - & 4 & 23 & 34 & 12 & - & - & - & 4 & - & 48 & 14 & 61 & 14 & 78 & 195 & - \\
\hline & Procloeo & - & - & 12 & 16 & 53 & 53 & - & - & 49 & 19 & - & 32 & - & 24 & - & 13 & - & - & - & 59 \\
\hline Ephemeridae & Ephemera & 26 & - & 38 & - & 46 & - & 43 & 19 & 16 & - & - & 23 & 32 & - & - & 49 & 13 & 89 & - & - \\
\hline \multirow[t]{2}{*}{ Ephemerellidae } & Ephemerella & 8 & - & - & 28 & - & 13 & 59 & - & - & - & 21 & - & - & 64 & 54 & - & - & - & 73 & 67 \\
\hline & Torleya & 6 & - & - & 33 & 14 & - & - & 23 & - & - & - & 16 & 45 & 45 & - & - & - & - & - & - \\
\hline enidae & Ecdyonurus & 14 & 48 & 64 & - & - & 17 & 14 & 24 & 17 & 24 & 39 & - & 26 & - & 12 & - & 64 & 14 & - & 45 \\
\hline \multirow{2}{*}{ Leptophlebiidae } & Paraleptophlebia & 4 & - & 24 & 16 & 9 & - & - & - & - & 25 & 37 & 44 & - & 10 & - & 1 & - & - & - & 12 \\
\hline & Habro & 18 & 14 & - & 4 & 3 & 19 & 4 & 37 & 14 & 39 & 22 & - & 12 & - & - & - & 55 & - & - & - \\
\hline \multicolumn{22}{|l|}{ Trichoptera } \\
\hline Lepistomatidae & & - & 11 & 46 & - & 79 & 63 & 43 & 19 & - & - & 8 & - & - & - & 48 & - & 74 & - & - & 52 \\
\hline midae & & 2 & - & 38 & 54 & 92 & - & 51 & - & 54 & 46 & - & 16 & - & 17 & - & 51 & - & 18 & - & - \\
\hline Rhyacophilidae & & 2 & 37 & - & 62 & - & 16 & - & 46 & 43 & 53 & - & - & 64 & 16 & 68 & - & - & - & 54 & - \\
\hline Polycentropodidae & & 4 & - & 22 & - & 84 & 8 & 19 & 73 & - & 43 & - & 16 & 33 & 12 & - & 24 & 94 & - & - & 25 \\
\hline Hydropsichidae & & 74 & 4 & - & 34 & - & 14 & - & 64 & 19 & - & 62 & 19 & - & - & 97 & - & 45 & - & 72 & - \\
\hline Limnephilidae & & 8 & - & 14 & 12 & - & - & 49 & - & 21 & 21 & 19 & 23 & 21 & 24 & 25 & - & - & - & 28 & 23 \\
\hline Hydroptilidae & & 6 & 59 & - & 19 & 33 & 9 & 53 & 59 & 13 & 19 & - & 4 & - & 18 & - & 67 & 40 & 24 & - & - \\
\hline \multirow{2}{*}{\multicolumn{2}{|c|}{$\begin{array}{l}\text { Isopoda } \\
\text { Asellidae }\end{array}$}} & 15 & 19 & & & & & & & & & & & 83 & & 431 & 207 & & & & \\
\hline & & 15 & 19 & 196 & 14 & - & 159 & 12 & - & 14 & 15 & 98 & 17 & 83 & 193 & 431 & 221 & 332 & 548 & 114 & 223 \\
\hline \multicolumn{2}{|l|}{ Amphipoda } & 74 & 12 & 638 & - & 24 & - & 54 & 48 & 112 & 224 & 57 & 137 & 224 & 118 & 328 & 347 & 115 & 789 & 521 & 114 \\
\hline Odonata & & & & & & & & & & & & & & & & & & & & & \\
\hline Calopterygidae & Calopteryx & 4 & 18 & 24 & 32 & - & 27 & 3 & 35 & 12 & 4 & 19 & - & - & 3 & 9 & - & 7 & - & - & - \\
\hline Corduliidae & & - & 5 & 18 & 1 & 8 & 7 & 9 & 15 & - & 21 & 32 & 27 & 6 & 2 & - & 2 & - & - & - & 1 \\
\hline Diptera & & & & & & & & & & & & & & & & & & & & & \\
\hline Athericidae & & 22 & - & 24 & - & 7 & - & 53 & - & 4 & - & 29 & - & 53 & - & - & 56 & - & 157 & - & 164 \\
\hline Ceratopogonidae & & 6 & 19 & - & 4 & - & 5 & - & 7 & - & 73 & 5 & 19 & - & 164 & - & 74 & 51 & - & - & - \\
\hline Chironomidae & & 162 & 94 & 118 & 14 & 9 & 12 & 56 & 79 & 9 & 44 & 97 & - & - & - & 189 & 46 & - & - & 224 & 449 \\
\hline Lim & & - & 73 & 18 & - & - & 19 & 54 & 45 & - & 9 & 14 & 23 & - & 125 & 256 & - & 46 & 45 & 118 & - \\
\hline Psicol & & 2 & - & 8 & 73 & 16 & - & 48 & - & - & 49 & 12 & 51 & 112 & - & 43 & 12 & - & - & 24 & - \\
\hline Simulida & & - & 46 & 48 & 51 & 23 & 15 & - & 84 & 56 & 23 & 24 & 46 & - & 89 & - & - & - & 18 & 32 & - \\
\hline Stratiomidae & & 4 & - & 8 & 23 & - & 16 & 75 & 73 & 15 & 18 & 16 & - & - & 73 & 158 & - & 36 & 275 & - & 23 \\
\hline Tipulidae & & 2 & 4 & 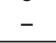 & 19 & 45 & 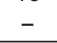 & - & 63 & 17 & - & 9 & 4 & 69 & - & 248 & - & - & - & - & 258 \\
\hline ligochaeta & & & & & & & & & & & & & & & & & & & & & \\
\hline . & & - & 5 & 6 & 1 & - & 3 & 2 & 4 & - & 4 & 9 & - & 4 & - & 12 & 14 & - & - & - & - \\
\hline Lumbricida & & 4 & 5 & 10 & - & 31 & 9 & 2 & 3 & 5 & - & 31 & 8 & 7 & 24 & 26 & 3 & 2 & - & 8 & - \\
\hline Tubificidae & & 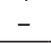 & 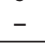 & 1 & 14 & . & 5 & 3 & 9 & - & 2 & 3 & - & 3 & - & 34 & 19 & 7 & 29 & 32 & 56 \\
\hline Coleoptera & & & & & & & & & & & & & & & & & & & & & \\
\hline & & - & 3 & 4 & - & 4 & 17 & 12 & 7 & 8 & 2 & 5 & - & 5 & - & 12 & 3 & - & - & 8 & 1 \\
\hline Dyt & & 4 & - & - & 5 & 3 & - & 12 & - & - & 2 & 7 & 3 & - & 9 & - & 7 & 6 & 15 & - & - \\
\hline Elminthidae & & 56 & 4 & 34 & 5 & - & 12 & - & 9 & 6 & 4 & 26 & 4 & 6 & 8 & 14 & 1 & - & 15 & - & 12 \\
\hline Girinidae & & 2 & 2 & 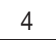 & 8 & 4 & 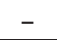 & 4 & 6 & 4 & 2 & 7 & 2 & 6 & 10 & - & 1 & 12 & - & - & 6 \\
\hline Hemiptera & & & & & & & & & & & & & & & & & & & & & \\
\hline Hydrol & & - & 3 & 4 & 2 & - & - & 5 & - & 3 & - & 3 & 6 & - & 7 & - & 2 & - & 4 & - & - \\
\hline Notonectidae & & 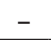 & - & 10 & - & 9 & - & - & 7 & 4 & 4 & 2 & - & 4 & - & 3 & 1 & - & - & 1 & 1 \\
\hline Megaloptera & & & & & & & & & & & & & & & & & & & & & \\
\hline Sialidae & Sialis & 4 & - & - & 6 & - & - & - & 7 & - & - & 9 & - & 1 & - & - & 4 & - & - & - & - \\
\hline Mollusca & & & & & & & & & & & & & & & & & & & & & \\
\hline Planorbidae & & 19 & - & 8 & 19 & 4 & 33 & - & 14 & 5 & - & 32 & - & 7 & 5 & 22 & 9 & 21 & 79 & 63 & - \\
\hline Lymneidae & & 4 & 44 & 24 & 14 & 19 & - & 13 & 12 & - & 7 & 39 & 5 & 6 & - & 34 & 22 & - & - & 45 & 94 \\
\hline Acarina & & 20 & - & 48 & - & - & - & - & - & - & - & 31 & - & 2 & - & 4 & 2 & 3 & - & 3 & - \\
\hline Ostracoda & & - & - & 32 & 2 & - & 1 & 3 & 4 & - & 6 & 3 & 2 & 7 & 9 & 12 & - & 3 & 4 & 4 & $\overline{1}$ \\
\hline Nematomorpha & & - & - & 4 & - & 3 & 3 & - & - & 2 & 1 & 1 & - & - & 1 & 1 & - & 3 & - & 1 & - \\
\hline E.B.I. class & & I & 1 & I & I & I & I & I & I & I & I & I & I & II & II & II & II & II & III & III & III \\
\hline
\end{tabular}




\section{Table III}

Faunal list of the main invertebrate organisms found in all sites.

Tableau III

Listes faunistiques des principaux de macroinvertébrés trouvés dans les stations étudiées.

Most sites had a substrate of rocks and some had a mix of sand and rocks. All sites offered suitable shelter for crayfish. In every watercourse, the riparian vegetation showed the typical arboreous and herbaceous species (Populus spp., Salix spp., Pedasytes hybrida, Veronica beccabunga, etc.). Invasive species, Rubus ulmifolius and Urtica dioica, occurred abundantly in deteriorated habitat (Aniene, Turano, Salto and Licenza River). Moreover, bryophytes occurred in environments that showed either no or moderate degrees of pollution, while these species were absent in more polluted sites.

\section{Macrobenthos community}

Table III shows the invertebrate communities observed in each site, and in particular, the number of specimens per every taxon. Moreover, the last line shows the quality class values of the Extended Biotic Index.

\section{Freshwater crayfish populations}

A total of 20 sites were monitored. Crayfish occurred in 17 sites and in at least 10 of these some individuals were caught in every sampling session. The total number of crayfish and the sex ratio (female number divided by total caught specimens) of each population are shown in Table IV.

Size-frequency distributions for each crayfish populations are shown in Figure 2; the length-frequency diagrams were represented using the total number of crayfishes caught during the four sampling sessions (in ordinate axis) and the post-orbital length values, in $1 \mathrm{~mm}$ intervals (in abscissa axis). Table $V$ presents catch per unit effort (number of individuals caught divided by the time spent sampling) and the weighted average of the biomass for every site. These results demonstrate the differences in CPUE and relative abundance between sites inserted in different water quality classes.

\section{Table IV}

Number of the individuals caught during the research period and sex-ratio of every monitored population.

\section{Tableau IV}

Nombre d'individus capturés pendant la période de recherche et sex-ratio des populations étudiées.

\begin{tabular}{|c|c|c|c|c|c|c|c|c|c|c|c|c|c|c|c|c|c|}
\hline & 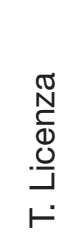 & 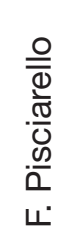 & 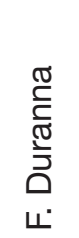 & 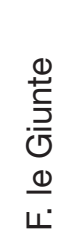 & 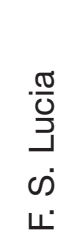 & 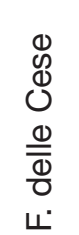 & $\begin{array}{l}\frac{0}{0} \\
\stackrel{0}{0} \\
\frac{0}{0} \\
\overline{0} \\
0 \\
\sqcup\end{array}$ & 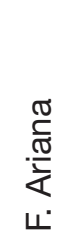 & 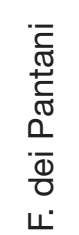 & 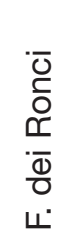 & 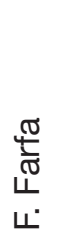 & $\begin{array}{l}\text { O্ } \\
\text { d } \\
\text { ஸे } \\
\frac{0}{\tilde{I}}\end{array}$ & 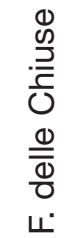 & 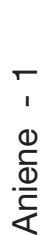 & 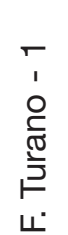 & 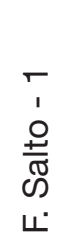 & 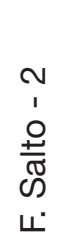 \\
\hline $\mathrm{n}^{\circ}$ ind. & 302 & 310 & 350 & 326 & 293 & 323 & 357 & 335 & 302 & 306 & 3 & 166 & 134 & 1 & 14 & 5 & 7 \\
\hline $\begin{array}{l}\text { sex ratio } \\
{[\mathrm{f} /(\mathrm{f}+\mathrm{m})]}\end{array}$ & 0,5 & 0,6 & 0,6 & 0,5 & 0,5 & 0,7 & 0,6 & 0,7 & 0,5 & 0,5 & 0 & 0,4 & 0,4 & 0 & 0,1 & 0,2 & 0,3 \\
\hline
\end{tabular}



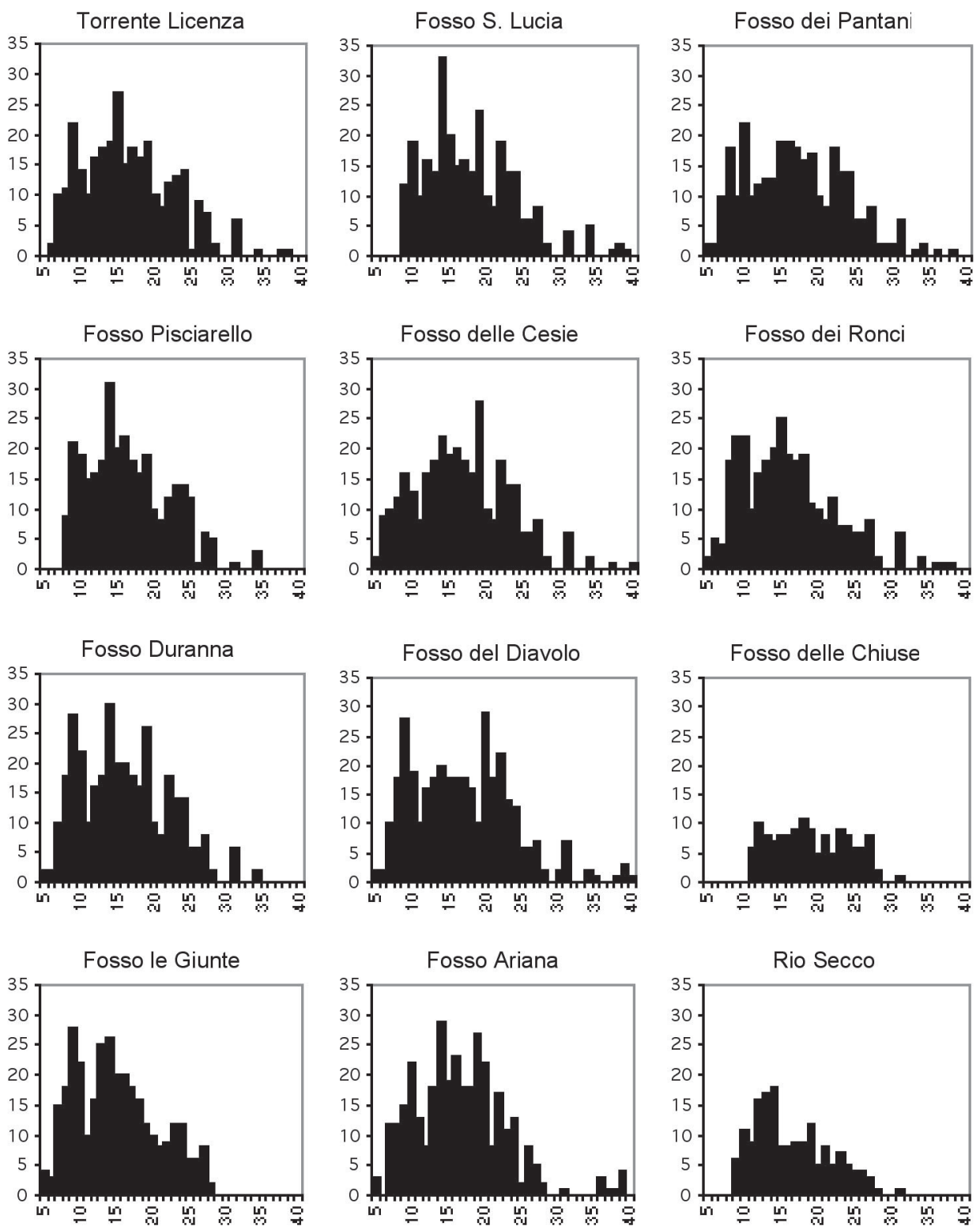

Figure 2

Length-frequency diagrams of the crayfish populations with more than 100 individuals (POL in abscissa axis; number of individuals in ordinate axis).

\section{Figure 2}

Diagramme longueur-fréquence des populations d'écrevisse de plus de 100 individus (POL en abscisse ; Nombre d'individus en ordonnée).

\section{DISCUSSION AND CONCLUSION}

These preliminary results show that the presence of white-clawed freshwater crayfish is not necessarily limited to watercourses with good environmental integrity, though they seem to be absent from polluted or deteriorated rivers. However, the presence/absence of a species does not provide all the information necessary to understand the environmental 
stress grade of a watercourse. The population structure of $A$. italicus can offer more detailed information on the environmental status and water quality.

During the research period, well-structured populations were found in watercourses that fall within class I of E.B.I., with the exception of Fosso delle Chiuse (class I), Fiume Farfa (class I) and Rio Secco (class II). In Fiume Farfa, the white-clawed crayfish is presumed not to have historically occurred, probably due to a lack of shelter, although one specimen was captured in July 2003. Fosso delle Chiuse revealed a population with a different structure compared to other crayfish populations with a class I E.B.I. designation, probably due to over-exploitation by local poachers. The white-clawed crayfish does not seem to find favourable environmental conditions in rivers that come within the second or third class of the Extended Biotic Index.

Nonetheless, it is possible to find them in these watercourses probably due to dispersal from more favourable upstream habitats. Rio Secco (class II), with moderate symptoms of deterioration, reveals a relatively good structured population, probably due

\section{Table V}

Average values of the catch per unit effort and relative abundance of the studied freshwater crayfish stocks.

\section{Tableau V}

Valeurs moyennes des captures par unité d'effort et abondance relative des stocks d'écrevisses étudiées.

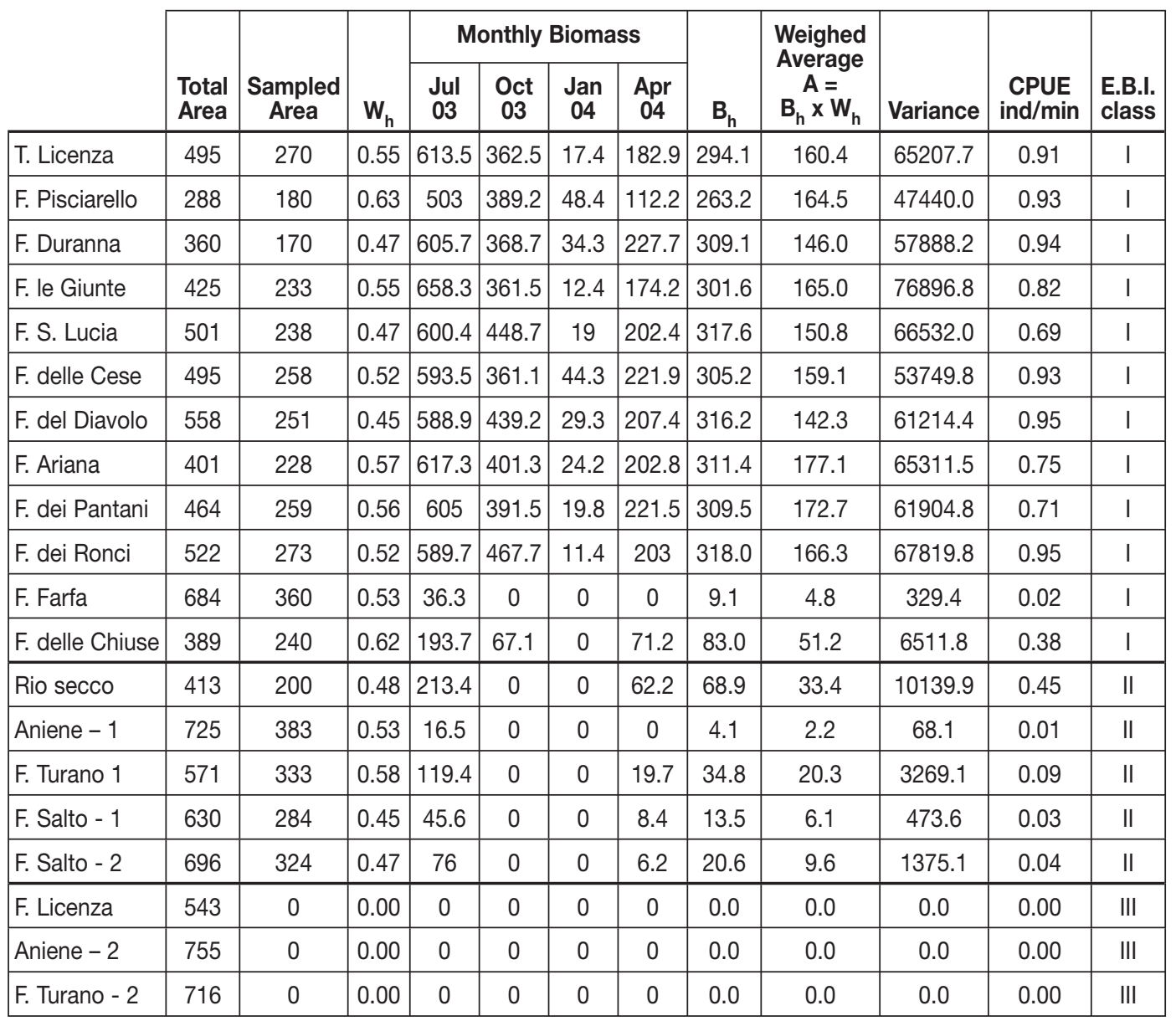


to high oxygen concentration, which increases crayfish tolerance to organic and pollutant substances.

Where the species historically occurs and tolerates extreme oxygen conditions, the white-clawed crayfish can be used as a "regional" bioindicator in combination with more broadly applicable assessment tools.

Illegal fishing affects several rivers in Latium, as it is reflected in the low number of large-sized crayfish. Poaching and bad exploitation of freshwater reduce the carrying capacity of the crayfish populations that live exclusively in very sensitive environments near the river source. White-clawed crayfish are well adapted to habitat conditions of these river tracts, which are affected by the arid Mediterranean climate. Indeed, unlike other geographic regions (REYNOLDS, 1979, 1982; LAURENT, 1988; ANTÓN et al., 2000; RALLO and GARCÍA-ARBERAS, 2000), in Central Italy A. italicus does not live in lentic waters, though it is possible to observe some freshwater crayfish populations in small glacial lakes over $1100 \mathrm{~m}$ a.s.l. The low genetic variability of these populations in this region (SANTUCCl et al., 1997) makes $A$. italicus more vulnerable and threatened.

For this reason, A. italicus should be included in monitoring projects, for its own preservation, but also because crayfish are very important in the trophic dynamics of freshwater environments and therefore act as a monitoring instrument for the management of freshwater communities. Given that one of the characteristics proper of bioindicators, i.e. wide distribution (FÜREDER and REYNOLDS, 2003), is not fulfilled, it is not possible to consider $A$. italicus meridionalis as a biological indicator. Its presence depends on biological, historical and human factors (BARBARESI and GHERARDI, 1997; ENGLUND and KLUPA, 2000; RALLO and GARCIA-ARBERAS, 2002; FUREDER et al., 2003) that constitute disadvantages in using this species as a bioindicator.

In Italy, the preliminary results of this research provide preliminary data concerning the relation between water quality and the status of crayfish populations. In Europe, many researchers consider $A$. italicus to be a sensitive species to pollutant substances and rapid changes of environmental conditions. However, a number of studies suggest that A. italicus can live in brooks with sub-optimal water quality. GRANDJEAN et al. (2000a, 2001) and BROQUET et al. (2002) had already demonstrated this phenomenon in France, and DEMERS and REYNOLDS (2002) in Ireland. Crayfish are necessarily dependent on specific features of the aquatic ecosystem. Habitat heterogeneity, water quality and lack of pollutants have been shown to enhance the survival of indigenous crayfish (FÜREDER et al., 2003; GRANDEJEAN et al., 2003; TROUILHE et al., 2003). However, differently from what observed in this research, there are also examples showing the presence of crayfish in environmental conditions ranging from natural to degraded levels (DEMERS et al., 2003; FÜREDER et al., 2003).

In a conservation fauna management context, animal species can be used as surrogate species (CARO and O'DOHERTY, 1999) to indicate various types of anthropogenic impacts, to track population changes of other species, to locate areas of high diversity, or to act as umbrella or flagship species. Due to its features and peculiarities, freshwater crayfish can be considered a good example of surrogate species (FÜREDER et al., 2003). Crayfish can act as umbrella or flagship species while their value as bioindicator is still debated, probably due to the lack of knowledge of combined factors responsible for their occurrence and for their population structure (FÜREDER and REYNOLDS, 2003).

Based on its ecological characteristics, freshwater crayfish can be used to monitor the lotic water quality in some regions and to integrate other more laborious and intensive tools, useful to understand and to predict environmental changes. In this context, the use of $A$. italicus meridionalis for the assessment of the environmental quality should be completed with other biotic indices or other managing instruments. The management 
of crayfish resources requires the preservation of both this species and the inland water habitat where they are found. Conservation projects are to be applied following the actual national and regional laws and their improvements.

\section{REFERENCES}

ALDERMANN D.J., POLGASE J.L., 1988. Pathogens, parasites and commensals. In: Holdich D.M. and Lowery R.S. (eds.), Freshwater Crayfish: Biology, Management and Exploitation, 167-212, Croom Helm, London.

ANTÓN A., SERRANO T., ANGULO E., FERRERO G., RALLO A., 2000. The uses of two species of crayfish as environmental quality sentinels: the relationship between heavy metal content, cell and tissue biomarker and physico-chemical characteristics of the environment. The Science of the Total Environment, 247, 239-251.

BARBARESI S., GHERARDI F., 1997. Italian freshwater decapods: exclusion between the crayfish Austropotamobius pallipes (Faxon) and the crab Potamon fluviatile (Herbst). Bull. Fr. Pêche Piscic., 347, 731-747.

BROQUET T., THIBAULT M., NEVEU A., 2002. Distribution and habitat requirements of the white-clawed crayfish, Austropotamobius pallipes, in a stream of the Pays de Loire region, France: an experimental and descriptive study. Bull. Fr. Pêche Piscic., 367, 717-728.

CAMPAIOLI S., GHETTI P.F., MINELLI A., RUFFO S., 1994. Manuale per il riconoscimento dei macroinvertebrati delle acque dolci italiane. Ed. Museo di Storia Naturale di Trento, vol. 1, $357 \mathrm{p}$.

CAMPAIOLI S., GHETTI P.F., MINELLI A., RUFFO S., 1998. Manuale per il riconoscimento dei macroinvertebrati delle acque dolci italiane. Ed. Museo di Storia Naturale di Trento, vol. 2, $135 \mathrm{p}$.

CARO T.M., O'DOHERTY G., 1999. On the use of surrogate species in conservation biology. Conservation Biology, 13, 805-814.

CHASEMARTIN C., 1967. Contribution à l'étude de l'économie calcique chez les astacidae. Thèse de doctoral de Sciences Naturelles. Archives Originales du Centre de Documentation du CNRS, Poitiers.

COVICH A.P., PALMER M.A., CROWL T.A., 1999. The role of benthic invertebrate species in freshwater ecosystems. BioScience, 49, 119-127.

DEMERSA., REYNOLDSJ.D.,2002. Asurvey of the white-clawed crayfish, Austropotamobius pallipes (Lereboullet), and of water quality in two catchments of Eastern Ireland. Bull. Fr. Pêche Piscic., 367, 729-740.

DEMERS A., REYNOLDS J.D., CIONI A., 2003. Habitat preference of different size class of Austropotamobius pallipes in an Irish river. Bull. Fr. Pêche Piscic., 370-371, 127137.

ENGLUND G., KLUPA J.J., 2000. Habitat use by crayfish in stream pools: influence of predators, depth and body size. Freshwater Biol., 43, 75-83.

FOSTER J., TURNER C., 1993. Toxicity and field simulated farm waste episodes to the crayfish Austropotamobius pallipes (Lereboullet): elevated ammonia and reduced dissolved oxygen concentrations. Freshwater Crayfish, 9, 249-258.

FRATINI S., ZACCARA S., BARBARESI S., GRANDJEAN F., SOUTY-GROSSET C., CROSA G., GHERARDI F., 2005. Phylogeography of the threatened crayfish (genus Austropotamobius) in Italy: implications for its taxonomy and conservation. Heredity, 94, 108-118. 
FÜREDER L., OBERKOFLER B., HANEL R., LEITER J., THALER B., 2003. The freshwater crayfish Austropotamobius pallipes in South Tyrol: heritage species and bioindicator. Bull. Fr. Pêche Piscic., 370-371, 79-95.

FÜREDER L., REYNOLDS J.D., 2003. Is Austropotamobius pallipes a good bioindicator? Round table discussion, Craynet meeting, Kilkenny, Ireland, 22-24 June 2003. Bull. Fr. Pêche Piscic., 370-371, 157-163.

GHERARDI F., HOLDICH D.M., 1999. Crayfish in Europe as Alien Species. How to Make the Best of a Bad Situation? A.A. Balkema, Rotterdam.

GRANDJEAN F., ROMAIN D., AVILE-ZARZA C., BRAMARD M., SOUTY-GROSSET C., MOCQUARD J.P., 1997. Morphometry, sexual dimorphism and size at maturity of the white-clawed crayfish Austropotamobius pallipes pallipes (Lereboullet) from a wild French population at Deux-Sèvres (Decapoda, Astacidea). Crustaceana, 70(1), 31-44.

GRANDJEAN F., GOUIN N., FRELON M., SOUTY-GROSSET C., 1998. Genetic and morphological systematic studies on the crayfish Austropotamobius pallipes (Decapoda: Astacidae). Journal of Crustacean Biology, 18, 549-555.

GRANDJEAN F., CORNAULT B., ARCHAMBAULT S., BRAMARD M., OTREBSKY G., 2000a. Life history and population biology of the white-clawed crayfish, Austropotamobius pallipes pallipes, in a brook from the Poitou-Charentes region (France). Bull. Fr. Pêche Piscic., 356, 055-070.

GRANDJEAN F., HARRIS D.J., SOUTY-GROSSET C., CRANDALL K.A., 2000b. Systematics of the European endangered crayfish species Austropotamobius pallipes (Decapoda: Astacidae). Journal of Crustacean Biology, 20, 522-529.

GRANDJEAN F., JOUTEX R., ROPIQUET A., BACHELIER E., BRAMARD M., 2001. Biological water quality assessment in running waters sites harbouring the endangered crayfish, Austropotamobius pallipes based on macroinvertebrates and its potential in habitat selection for restocking. Freshwater Crayfish, XIII, 338-348.

GRANDJEAN F., BOUCHON D., SOUTY-GROSSET C., 2002a. Systematic of the European endangered crayfish species Austropotamobius pallipes (Decapoda: Astacidae) with a re-examination of the status of Austropotamobius berndhauseri. J. Crustacean Biol., 22(3), 677-681.

GRANDJEAN F., FRELON-RAIMOND M., SOUTY-GROSSET C., 2002b. Compilation of molecular data for the phylogeny of the Austropotamobius: one species or several? Bull. Fr. Pêche Piscic., 367, 671-680.

GRANDJEAN F., MOMON J., BRAMARD M., 2003. Biological water quality assessment of the white-clawed habitat based on macroinvertebrate communities: usefulness for its conservation. Bull. Fr. Pêche Piscic., 370-371, 115-125.

GROOMBRIDGE B., 1993. 1994 IUCN Red List of Threatened Animals, IUCN, Gland.

HOGGER J.B., LOWERY R.S., 1982. The encouragement of freshwater crayfish populations by the attention to the construction and maintenance of waterways. J. Inst. Water Engnrs \& Scient., 36, 214-220.

HOLDICH D.M., LOWERY R.S., 1988. Freshwater Crayfish: Biology, Management and Exploitation. Croom Helm, London, 498 p.

HOLDICH D.M., REEVE I.D., 1991. The distribution of freshwater crayfish in the British Isles with particular reference to crayfish plague, alien introductions and water quality. Aquatic Conservation, 1(2), 139-158.

HOLDICH D.M., ROGERS W.D., REYNOLDS J.D., 1999. Crayfish in British Isles. In: GHERARDI F. and HOLDICH D.M. (eds.), Crayfish in Europe as Alien Species. How to make the best of a bad situation? 221-235. A.A. Balkema, Rotterdam. 
LARGIARDER C.R., HERGER F., LÖRTSCHER M., SCHOLL a., 2000. Assessment of natural and artificial propagation of the white-clawed crayfish (Austropotamobius pallipes species complex) in the Alpine region with nuclear and mitochondrial markers. Molecular Ecology, 9, 25-37.

LAURENT P.J., 1988. austropotamobius pallipes and A. torrentium, with observations on their interactions with other species in Europe. In: HOLDICH D.M. and LOWERY R.S. (eds.), Freshwater Crayfish: Biology, Management and Exploitation, 341-364, Croom Helm, London.

LOWERY R.S., HOGGER J.B., 1986. The effects of river engineering and disease on a population of Austropotamobius pallipes in the river Lea, UK. Freshwater Crayfish, 6, 94-99.

MANCINI L., ARCÁ G., 2000. Carta della Qualità Biologica dei Corsi d'Acqua della Regione Lazio. Regione Lazio. Assessorato Ambiente. Dipartimento Ambiente e Protezione Civile, $156 \mathrm{p}$.

MOMOT W.T., 1995. Redefining the role of crayfish in aquatic ecosystems. Reviews in Fisheries Science, 3, 33-36.

RALLO A., GARCÍA-ARBERAS L., 2000. Population structure and dynamics and habitat conditions of the native crayfish Austropotamobius pallipes in a pond: a case study in Basque Country (Northern Iberian Peninsula). Bull. Fr. Pêche Piscic., 356, 005016.

RALLO A., GARCÍA-ARBERAS L., 2002. Differences in abiotic water conditions between fluvial reaches and crayfish fauna in some northern rivers of Iberian Peninsula. Aquat. Living Resour., 15, 119-128.

REYNOLDS J.D., 1979. Crayfish ecology in Ireland. Freshwater Crayfish, 4, 215-220.

REYNOLDS J.D., 1982. Notes on the Irish distribution of the freshwater crayfish. Bulletin of the Irish Biogeography Society, 6, 18-24.

REYNOLDS J.D., GOUIN N., PAIN S., GRANDJEAN F., DEMERS A., SOUTY-GROSSET C., 2002. Irish crayfish populations: ecological survey and preliminary genetic findings. Freshwater Crayfish, 13, 584-594.

SANTUCCI F., IACONELLI M., ANDREANI P., CIANCHI R., NASCETTI G., BULLINI L., 1997. Allozyme diversity of European freshwater crayfish of the genus Austropotamobius. Bull. Fr. Pêche Piscic., 347, 663-676.

SCALICI M., 2004. Distribution, ecology, structure and dynamic of population, reproductive biology and proposal of managing and conservation of the freshwater crayfish Austropotamobius italicus (Decapoda: Astacidae) in the water system of Latium. $\mathrm{PhD}$ thesis, $108 \mathrm{p}$.

SCALICI M., VIGNOLI L., GIBERTINI G., 2004. Observation in the diet habit of the autochthonous freshwater crayfish, Austropotamobius pallipes italicus (Faxon). Biologia Ambientale, 18(1), 51-56.

SPEDICATO M.T., CANNAS A., 2000. La pesca responsabile. In: CATAUDELLA S. and CARRADA G.C., Un mare di risorse. Introduzione alla conservazione ed alla gestione delle risorse ittiche, 67-139.

TROUILHE M.C., RICARD F., PARINET B., GRANDJEAN F., SOUTY-GORSSET C., 2003. Management of the white-clawed crayfish (Austropotamobius pallipes) in Western France: abiotic and biotic factors study. Bull. Fr. Pêche Piscic., 370-371, 97-114.

WESTMAN K., 1985. Effects of habitat modification on freshwater crayfish, In: Habitat modifications and freshwater fisheries, ALABASTER J.S. ed., Butterworths, London, 245-255. 
